\title{
Genome Sequence, Transcriptome, and Annotation of Rodent Malaria Parasite Plasmodium Yoelii Nigeriensis N67
}

\section{Cui Zhang}

National Institutes of Health

\section{Cihan Oguz}

National Institutes of Health

\section{Sue Huse}

National Institutes of Health

Lu Xia

National Institutes of Health

Jian Wu

National Institutes of Health

\section{Yu-Chih Peng}

National Institutes of Health

\section{Margaret Smith}

National Institutes of Health

Jack Chen

National Institutes of Health

Carole A. Long

National Institutes of Health

Justin Lack

National Institutes of Health

Xin-zhuan Su ( $\nabla$ xsu@niaid.nih.gov)

National Institutes of Health

\section{Research Article}

Keywords: Plasmodium, mouse, DNA sequence, transcript, proteome, polymorphism

Posted Date: February 4th, 2021

DOl: https://doi.org/10.21203/rs.3.rs-162427/v1 
License: (c) (i) This work is licensed under a Creative Commons Attribution 4.0 International License. Read Full License

Version of Record: A version of this preprint was published at BMC Genomics on April 26th, 2021. See the published version at https://doi.org/10.1186/s12864-021-07555-9. 
1 Genome sequence, transcriptome, and annotation of rodent malaria parasite

$2 \quad$ Plasmodium yoelii nigeriensis $\mathbf{N 6 7}$

3

4 Cui Zhang ${ }^{1 *}$, Cihan Oguz ${ }^{2 *}$, Sue Huse $^{2}$, Lu Xia $^{1,3}$, Jian Wu ${ }^{1}$, Yu-Chih Peng ${ }^{1}$, Margaret Smith ${ }^{1}$, 5 Jack Chen ${ }^{4}$, Carole A. Long ${ }^{1}$, Justin $\mathrm{Lack}^{2}$, and Xin-zhuan $\mathrm{Su}^{1 \#}$

$7 \quad{ }^{1}$ Malaria Functional Genomics Section, Laboratory of Malaria and Vector Research, National

8 Institute of Allergy and Infectious Disease, National Institutes of Health, Bethesda, MD 20892-

9 8132, USA;

$10 \quad{ }^{2}$ NIAID Collaborative Bioinformatics Resource (NCBR), Frederick National Laboratory for

11 Cancer Research, Leidos Biomedical Research, Inc., Frederick, MD 21702, USA

$12{ }^{3}$ State Key Laboratory of Medical Genetics, Xiangya School of Medicine, Central South

13 University, Changsha, Hunan 410078, The People's Republic of China.

$14{ }^{4}$ The NCI sequencing facility, 8560 Progress Drive, Room 3007, Frederick Md 21701, USA

15

16 'Co-first authors

$17 \quad$ \#Correspondence address to: Xin-zhuan Su at xsu@niaid.nih.gov

19 Short title: $P$. yoelii nigeriensis genome and transcriptome 


\section{Abstract}

25 Background: Rodent malaria parasites are important models for studying host-malaria parasite

26 interactions such as host immune response, mechanisms of parasite evasion of host killing, and

27 vaccine development. One of the rodent malaria parasites is Plasmodium yoelii, and multiple $P$.

28 yoelii strains or subspecies that cause different disease phenotypes have been widely employed

29 in various studies. The genomes and transcriptomes of several $P$. yoelii strains have been

30 analyzed and annotated, including the lethal strains of Plasmodium y. yoelii YM (or 17XL) and

31 non-lethal strains of Plasmodium y. yoelii 17XNL/17X. Genomic DNA sequences and cDNA

32 reads from another subspecies $P . y$. nigeriensis $\mathrm{N} 67$ have been reported for studies of genetic

33 polymorphisms and parasite response to drugs, but its genome has not been assembled and

34 annotated.

35 Results: We performed genome sequencing of the N67 parasite using the PacBio long-read

36 sequencing technology, de novo assembled its genome and transcriptome, and predicted 5,383

37 genes with high overall annotation quality. Comparison of the annotated genome of the N67

38 parasite with those of YM and 17X parasites revealed a set of genes with N67-specific orthology,

39 expansion of gene families, particularly the homologs of the Plasmodium chabaudi erythrocyte

40 membrane antigen, large numbers of SNPs and indels, and proteins predicted to interact with

41 host immune responses based on their functional domains.

42 Conclusions: The genomes of N67 and 17X parasites are highly diverse, having approximately

43 one polymorphic site per 50 base pairs of DNA. The annotated N67 genome and transcriptome

44 provide searchable databases for fast retrieval of genes and proteins, which will greatly facilitate

45 our efforts in studying the parasite biology and gene function and in developing effective control

46 measures against malaria. 
47 Key words: Plasmodium, mouse, DNA sequence, transcript, proteome, polymorphism

\section{Background}

50 Malaria is one of the deadly tropical infectious diseases that impacts the health of hundreds of

51 millions of people [1]. The lack of an effective vaccine, emergence of drug resistant parasites

52 and insecticide resistant mosquitoes, and incomplete understanding of the disease mechanisms

53 are the major factors that impede disease control and elimination. Vaccine development and in

54 depth studies of disease molecular mechanisms using human populations are limited by ethical

55 regulations and relatively high costs. Animal disease models such as parasites infecting rodents

56 and non-human primates are important systems for studying malaria and have been widely used

57 for vaccine development and for studying the molecular mechanisms of host-parasite interaction

$58[2,3]$. Of course, results obtained from animal models need to be verified in human infection

59 because there are differences in disease mechanism due to variation in genetic backgrounds of

60 both the parasites and the hosts.

61

62 Plasmodium yoelii is one of the rodent malaria species that includes several parasite strains or

63 subspecies well-characterized genetically and phenotypically $[4,5]$. Some of the $P$. yoelii strains

64 are genetically diverse, whereas others are closely related or derived from a common ancestor

65 during laboratory passages in mice [4, 6, 7]. Mice infected with these $P$. yoelii strains generally

66 have dramatic differences in parasitemia, disease severity, pathology, and host immune response

67 [8]. For example, P. y. yoelii 17X (or 17XNL) and P. y. yoelii 17XL (or YM) are closely related

68 parasites genetically. Indeed, these parasites were derived from a parasite isolated from a wild

69 thicket rat in the Central African Republic [7]. 17XL and YM lines became fast growing and 
70 lethal during passages of $17 \mathrm{X}$ parasites in mice in two separate laboratories, whereas $17 \mathrm{X}$ or

71 17XNL remained slow growing and non-lethal [7]. These parasites also stimulate different host

72 responses and pathology [9-12]. Another example of parasites having closely related genomes

73 but with different virulence is the $P . y$. nigeriensis N67 and $P . y$. nigeriensis N67C parasite pair.

74 The genomes of N67 and N67C are very similar [5, 6]; however, they produce quite different

75 disease phenotypes in C57BL/6 mice. Infection of N67 stimulates a strong early type I interferon

76 (IFN-I) response, leading to a decline of parasitemia to below 5\% day 7 post infection (pi). The

77 parasitemia rebounds to 50-60\%, and the host dies at day 20 pi [13]. In contrast, mice infected

78 with N67C produce a strong T cell and INF- $\gamma$ mediated inflammatory responses and die day $7 \mathrm{pi}$

79 [14]. A C741Y amino acid substitution in the P. yoelii erythrocyte binding-like protein (PyEBL)

80 contributes to the differences in virulence and immune response, but other parasite genes also

81 play a role in the differences in disease phenotypes [15]. Identification of the genes or genetic

82 differences between N67 and N67C parasite will facilitate our understanding of the molecular

83 mechanisms of virulence and disease phenotypes in these infections.

85 With the advance of DNA sequencing technologies, the genomes and transcriptomes of many

86 rodent malaria parasites, including those of YM, 17X, and 17XNL strains, have been sequenced

87 and annotated [16-20]. The genomes of Plasmodium berghei and Plasmodium chabaudi parasites

88 are approximately $18.5-19 \mathrm{Mb}$, whereas the $P$. yoelii $\mathrm{YM}$ genome is $22.75 \mathrm{Mb}$ containing 5,675

89 predicted genes [18]. There are only eight genes with single nucleotide polymorphisms (SNPs)

90 detected between the genomes of the YM and 17X strains [18], supporting isogenic parasites

91 recently derived from the same ancestor [7]. Although RNA and DNA sequencing studies using

92 short Illumina reads from the N67 parasite have been previously carried out to investigate 
93 genetic polymorphisms and parasite response to drugs [6, 19], the N67 genome has not been

94 assembled and annotated, which impedes studies of the gene functions, parasite biology, and

95 virulence of the parasite. In this study, we sequenced the genome of the N67 parasite using

96 PacBio sequencing technology that produces long sequence reads, assembled, and annotated its

97 genome based on de novo assembled genome sequences and multiple transcriptomes.

98 Comparison of the N67 genome sequences with those of the YM and 17X parasites revealed a

99 set of proteins with N67-specific orthology, protein families predicted to regulate host immune

100 responses, expansion of critical gene families, and a large number of SNPs and indels that pass

101 stringent filtering criteria. These results have the potential to greatly facilitate our efforts in

102 studying the parasite biology and in developing effective control measures against malaria.

103

104 Results

105 Genome sequencing, read statistics, and de novo assembly of the parasite genomes

106 We prepared DNA samples for PacBio sequencing from the N67 parasite we obtained previously

107 [5]. Genomic DNAs were fragmented and sequenced on a PacBio Sequel using PacBio SMRT

108 cell long read technology [21]. The polymerase reads from sequencing machine were first

109 filtered to remove barcodes and low-quality sequences using the Hierarchical Genome Assembly

110 Process (HGAP) (Fig. S1a). We obtained 1,111,721 subreads consisting of 6,733,837,360 bp for

111 the N67 parasite, providing 233 mean coverages with an averaged barcode quality of 72 . The

112 longest subread length was $195,628 \mathrm{bp}$ and the mean read length was 70,695 bp. The subreads

113 were then assembled into 61,130 circular consensus sequencing (CCS) reads with a mean CCS

114 coverage of 13.5-fold for the parasite.

115 
116 We next de novo assembled the N67 CCS reads into 121 contigs consisting of 21,277,183 bp,

117 with the largest contigs being 979,279 bp (Table 1). For the assembled sequences, the N50 index

118 was 300,848 bp with $95.8 \%$ of the N67 sequences in contigs > $50 \mathrm{~kb}$ (Fig. S1b). The GC content

119 of the sequences for the parasites is $\sim 22 \%$ for the nuclear genome and $\sim 30 \%$ for mitochondrial

120 and the plastid genomes, similar to those of the 17X parasite.

a

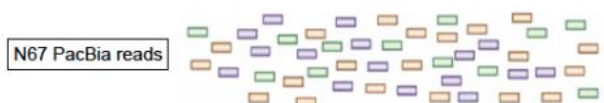
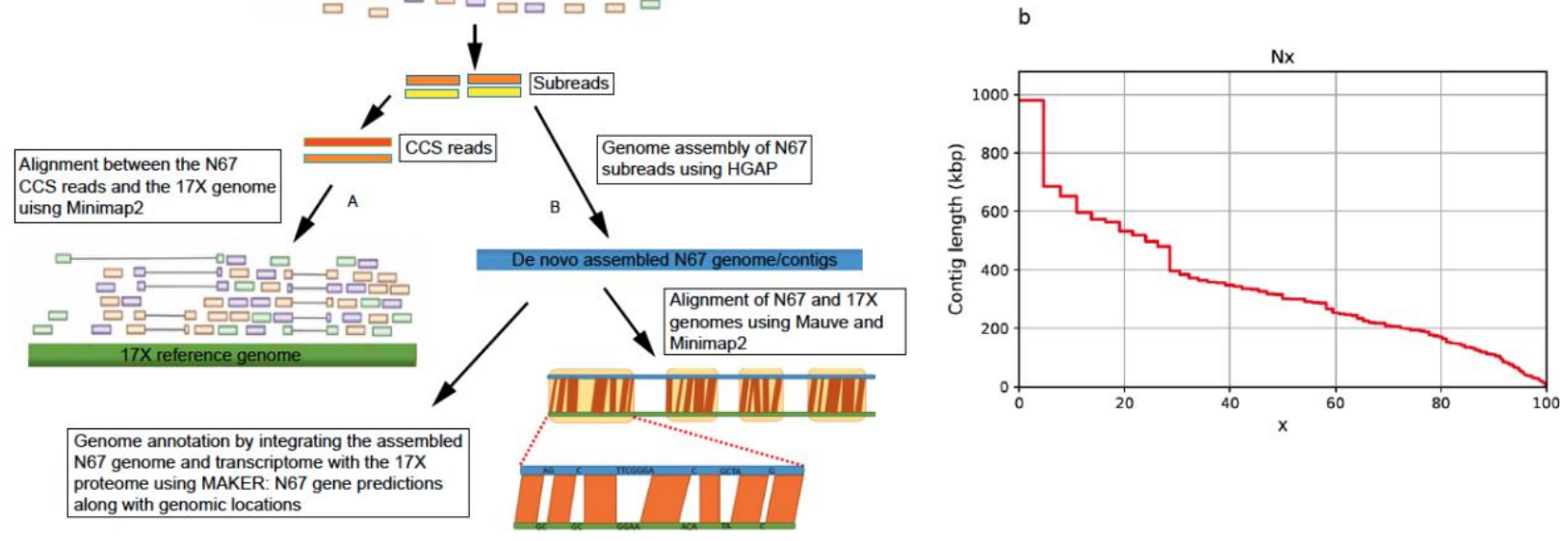

121

122 Fig. S1. Strageties of genome assembly and annotation, and plot of contig length distributions of

123 the Plasmodium y. yoelii N67 parasite genome assembly. a, Diagram illustrating the processes of

124 aligning N67 CCS reads (A) and contigs (B) to the 17X genomes. HGAP, Hierarchical genome

125 assembly process; CCS read, circular consensus sequencing read. b, Plot of contig length

126 distribution. The $\mathrm{X}$-axis is percentage of the contigs with lengths (base pair) greater than the

127 values indicated on the $\mathrm{Y}$-axis.

128

129 Alignment of N67 sequences to the $17 \mathrm{X}$ assembled genome

130 Before investigating the diversity of the N67 genome and performing genome annotation, we

131 aligned both the CCS reads and the assembled contigs to the updated 17X reference genome in

132 PlasmoDB, version 46 (https://plasmodb.org/plasmo/) [18, 22] using Minimap2 [23] and the 
133 progressiveMauve algorithm [24] that performs contig-by-contig alignment between the

134 assembly and the $17 \mathrm{X}$ reference (Fig. S1a). A total of $\sim 23 \mathrm{Mb}$ from the N67 CCS reads were

135 aligned to the 14 chromosomes of the $17 \mathrm{X}$ parasite, 34,324 bp to the plastid genome, and 6,083

136 bp to the mitochondrial genome, suggesting good genome coverages (Table 2). The mean CCS

137 read coverages were 11.0-13.7 for the autosomes, 43.9 for the plastid genome, and 334.3 for the

138 mitochondrial genome. In addition to the base-level alignment, we also aligned 101 of the 121

139 N67 contigs to the $17 \mathrm{X}$ reference genome using the progressiveMauve algorithm (Fig. 1)

140 and $18.1 \mathrm{Mb}$ of the 21.1 Mb (86\%) de novo assembled N67 genome to the 17X genome using

141 Minimap2. The low GC content of the parasite DNA and the abundance of low-complexity

142 repeats in the genomes pose challenges to the assembly process and the alignment of the

143 assembled N67 genome to the 17X reference. Therefore, approximately $14 \%$ of the N67

144 assembly did not align to the $17 \mathrm{X}$ genome at contig level.

145

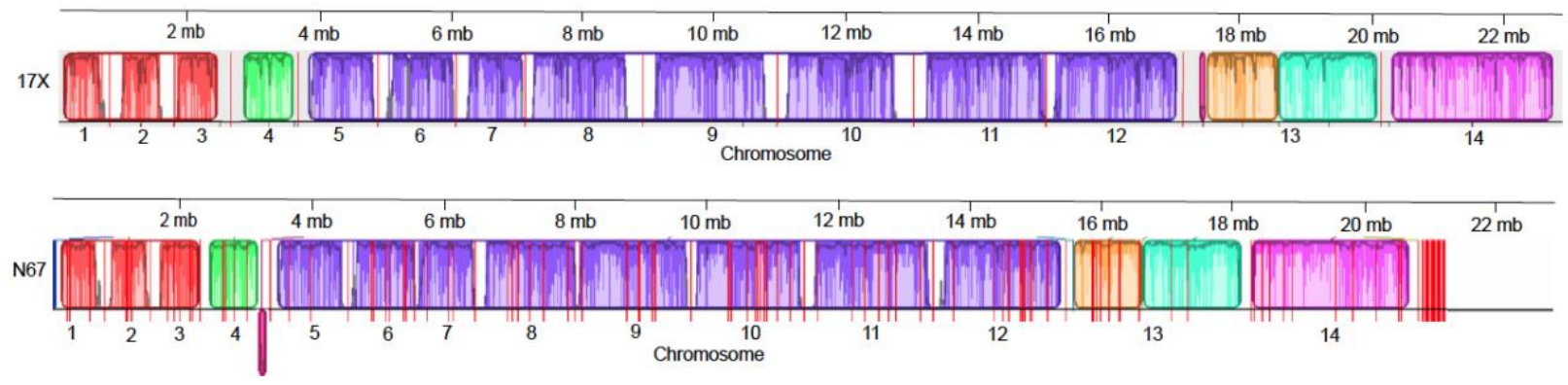

147 Fig. 1. Alignment of Hierarchical Genome Assembly Process (HGAP) assembled N67 contigs to

148 the $17 \mathrm{X}$ chromosomes. The alignments were generated using progressiveMauve. Each color

149 corresponds to a localized co-linear block (LCB) that is conserved across the two genomes.

150 Inside each LCB, the jagged dark lines represent the similarity profile; with darker colors

151 representing higher similarity regions. The vertical red lines indicate chromosome boundaries in 
$15217 \mathrm{X}$ and the contig boundaries on the N67 sequences. Note a contig on N67 chromosome 4 that

153 is inverted (presented under the chromosome line) in reference to that of $17 \mathrm{X}$ sequence.

155 RNA-Seq data and de novo transcriptome assembly of the $\mathrm{N67}$ parasite

156 To facilitate genome annotation and gene prediction, we also sequenced mRNA of blood stages

157 from eight mice infected with N67 Illumina sequencing method. Overall, 82.9\% of the RNA-Seq

158 reads from the samples uniquely mapped to the $17 \mathrm{X}$ genome and were retained for transcriptome

159 assembly, The majority of the remaining reads were either uniquely mapped to the mouse

160 genome (4.7\%) or did not map to any genomes of human, mouse, bacteria, fungi and virus

161 (9.3\%) based on results from the FastQ Screen [25]. The rest of 3.1\% of reads were mapped to

162 human, fungi, bacteria or multiple genomes. We then used Trinity [26] to perform de novo

163 transcriptome assembly and obtained 25,689 transcripts containing 39,856,633 bp with an

164 average GC content of $23.5 \%$ (Table S1). The N50 was 1,952 bp with the largest transcript

165 being $19,550 \mathrm{bp}$. The N67 Illumina reads were aligned to the de novo assembled $P$. yoelii

166 transcriptome using Bowtie2 [27], resulting in 95.4\% of the N67 read pairs concordantly aligned

167 to the assembled transcriptome, showing a high level of overall read support for the assembly.

169 Gene predictions and functional annotation

170 We predicted 5,383 genes/proteins from the N67 genome, including all the sequences not aligned

171 to the 17X genome, using the MAKER pipeline [28] as described in the Methods (Table S2). For

172 a high quality and well-annotated assembly, at least $90 \%$ of the predicted proteins are required to

173 have annotation edit distance (AED) values of less than 0.5 [28]. For the N67 proteome, $98 \%$

174 and 94\% had AED (base pair level) and eAED (exon level) values less than 0.5, respectively. 
175 Additionally, more than $50 \%$ of the proteome should ideally contain a recognizable protein

176 domain for a well-annotated proteome [28]. Ninety-two percent of the predicted N67 proteins

177 have recognizable domains and/or are assigned to protein families. Furthermore, the smallest

178 predicted N67 protein has 16 amino acids (N67_005372, Table S2), similar to the smallest

179 predicted protein of 15 amino acids in the 17X proteome. Search of N67_005372 protein

180 sequence (MRVNKYVSVNMKMNYT) against the 17X and YM proteome did not return any

181 hit; however, it has a 79\% sequence identity to serine hydroxymethyltransferase of

182 thermoacidophilic archaea Thermoplasma volcanium.

183

184 Search of the N67 proteome against InterPro database

185 (https://www.ebi.ac.uk/interpro/search/sequence/) of protein families, domains and functional

186 sites using InterProScan revealed that the largest five groups of proteins were the YIR antigens

187 (750 members), P-loop containing nucleoside triphosphate hydrolases (272), subtelomeric

188 PYST-A proteins (118), WD40-repeat-containing domain superfamily (89), and homologous

189 proteins of P. chabaudi erythrocyte membrane protein 1 (PcEMA1) (83) (Table S3).

190 Interestingly, the PcEMA1 was initially described from P. chabaudi parasites as an acidic

191 phosphoprotein that might modulate the structure of the red cell membrane to the advantage of

192 the parasite [29]. It has two tandem repats $(16 \times 8 \mathrm{AA}$ and $2 \times 9 \mathrm{AA})$ that may mediate genetic

193 recombination and gene member expansion possibly through microhomology-mediated end

194 joining (MMEJ) [30]. The expansion of this gene family in N67 parasites suggests that the

195 PcEMA1 proteins may play a role in interaction with host immune system. Some other

196 interesting groups included 43 proteins with DEAD/DEAH box helicase domain, 22 proteins

197 with AP2/ERF domain, and 7 proteins with Rh5 coiled-coil domain. 
198 We also searched the predicted N67 proteome for protein domains associated with pathways

199 within the Reactome pathway database. The top five largest Reactome groups were major

200 pathways of rRNA processing (122 proteins), regulation of expression of SLITs and ROBOs

201 (117), SRP-dependent cotranslational protein targeting to membrane (92), GTP hydrolysis and

202 joining of the 60S ribosomal subunit (91), and L13a-mediated translational silencing of

203 ceruloplasmin expression (89) (Table S4). Interestingly, there were also many proteins involved

204 in viral mRNA translation (78) and immune responses such as pathways of antigen processing

205 (64), neutrophil degranulation (36), NFאB activation in B cells (35), CLEC7A (Dectin-1)

206 signaling (35), downstream TCR signaling (35), FCERI mediated NFkB activation (35),

207 interleukin-1 signaling (35), NIK noncanonical NFאB signaling (35), and Vpu mediated

208 degradation of CD4 (35), TNFR2 non-canonical NFאB (34), and genes in MHC class II antigen

209 presentation (23) (Table S5). The molecules in the viral mRNA translation are mostly structural

210 constituents of ribosome proteins that are likely essential for the translation of parasite proteins.

211 Toxoplasma parasites secrete effector proteins into the host cell to co-opt host transcription

212 factors and modulate host immune responses [31]. Some of the proteins grouped with immune

213 response pathways could play important roles in regulating host immune response to the parasite

214 infection.

215

\section{Estimates of completeness of the N67 genome and transcriptome}

217 We next used Benchmarking Universal Single-Copy Orthologs (BUSCO 3.0.2) to assess the 218 completeness of the assembled N67 genome. Of the 3,642 Plasmodium and 446 Apicomplexa

219 BUSCO gene sets, 3,369 (92.5\%) and $431(96.6 \%)$ were present in the N67 genome assembly, 220 respectively (Table S6). We also evaluated the extent to which the assembled N67 transcriptome 
221 matched the BUSCO gene sets across the Apicomplexa and Plasmodium. Approximately $92.8 \%$

222 of the BUSCO Apicomplexa gene set and $71.3 \%$ of the Plasmodium gene set were present in the

223 assembled N67 transcriptome (Table S6). The N67 transcriptome genes matching the

224 Plasmodium BUSCO gene set included 1,448 complete and single-copy genes (39.8\%), 1,149

225 (31.5\%) complete and duplicated genes, and 338 fragmented sequences (9.3\%) (Table S6).

226 There were also 707 genes (19.4\%) missing from the Plasmodium BUSCO gene set; some of the

227 missed genes might not be expressed in the blood stages. The genomic sequences from PacBio

228 sequencing appear to provide more complete gene assembly than those from short Illumina RNA

229 seq data.

230

$231 P$. yoelii common orthogroups and putative proteins with N67-specific orthology

232 The N67 and 17X (or YM) parasites belong to two subspecies of P. yoelii, and the genomes of

233 these parasites are quite diverse $[4,6]$. It is potentially interesting to identify genes common and

234 unique (or highly diverse) in these parasite genomes. Therefore, we compared the 5,383

235 predicted proteins from N67 with 6,092 17X proteins and 5,685 YM proteins using OrthoFinder

236 [32] and identified a core set of 4,539 orthogroups shared among the N67, 17X, and YM

237 genomes (Fig. 2a). Out of a total 17,160 proteins from the three parasite strains, 17,035 (99.3\%)

238 were placed in 5,230 orthogroups based on searches of sequence similarity using DIAMOND

239 within the latest OrthoFinder framework [33, 34]. Of the 5,383 N67 proteins, 5,294 were

240 assigned to orthogroups, including 110 in 12 N67 specific orthogroups (Table S7 and Table S8).

241 There were also 89 proteins that could not be assigned to any orthogroup, leading to a total of

242199 proteins that appear to have N67-specific orthology. These proteins had slightly lower

243 pairwise bit-scores than those assigned to the common orthogroups (Fig. 2b). 
a

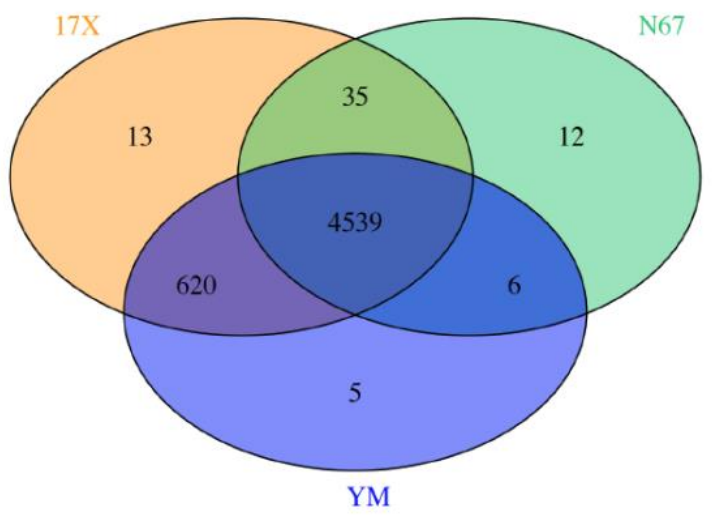

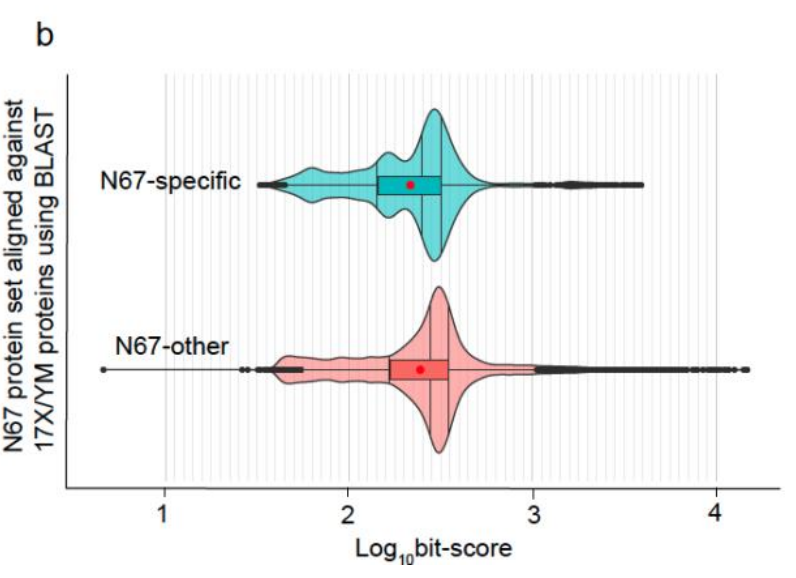

Fig. 2. Shared and strain-specific orthogroup counts identified from Plasmodium y. yoelii YM, $P$.

$246 y$. yoelii 17X, and $P$. y. nigeriensis N67 parasites using OrthoFinder [32]. a, Venn diagram of

247 shared and strain-specific orthogroups; $\mathbf{b}, \log _{10}$-transformed bit-score distributions for N67

248 proteins that are not assigned to any orthogroup plus those in N67-specific orthogroups (N67-

249 specific) and proteins assigned to orthogroups having at least one 17X or YM protein (N67-

250 other). The bit-scores are derived from pairwise BLAST alignments within the Orthofinder

251 framework, where all queries were N67 protein sequences that were aligned against the $17 \mathrm{X}$ and

252 YM sequences. The red dots indicate the mean values of bit-score distributions, whereas the

253 vertical lines within the violins indicate the median, upper and lower quartile values.

255 To further characterize N67-specific proteins, we used BLAST to align the 199 N67 proteins 256 against the $17 \mathrm{X}$ proteome and showed that the majority proteins had motifs matching members 257 of highly diverse gene families. Among the 199 proteins, 91 are hypothetical or uncharacterized 258 proteins, 64 are PIR/YIR proteins, 22 are Fam-A/B proteins, and five are reticulocyte binding 259 proteins (Table S9). Clustering the proteins based on sequence similarity generated three 260 dendrograms, one consisting of Fam-A and Fam-B proteins (Fig. S2a and Table S9), another 261 one consisting of 10 YIR proteins (Fig. S2b), and a third one of three subclusters of YIR proteins 
262 (Fig. S2c). The YIR proteins in cluster B and C are quite different and could not be clustered

263 together, suggesting potentially different origins.
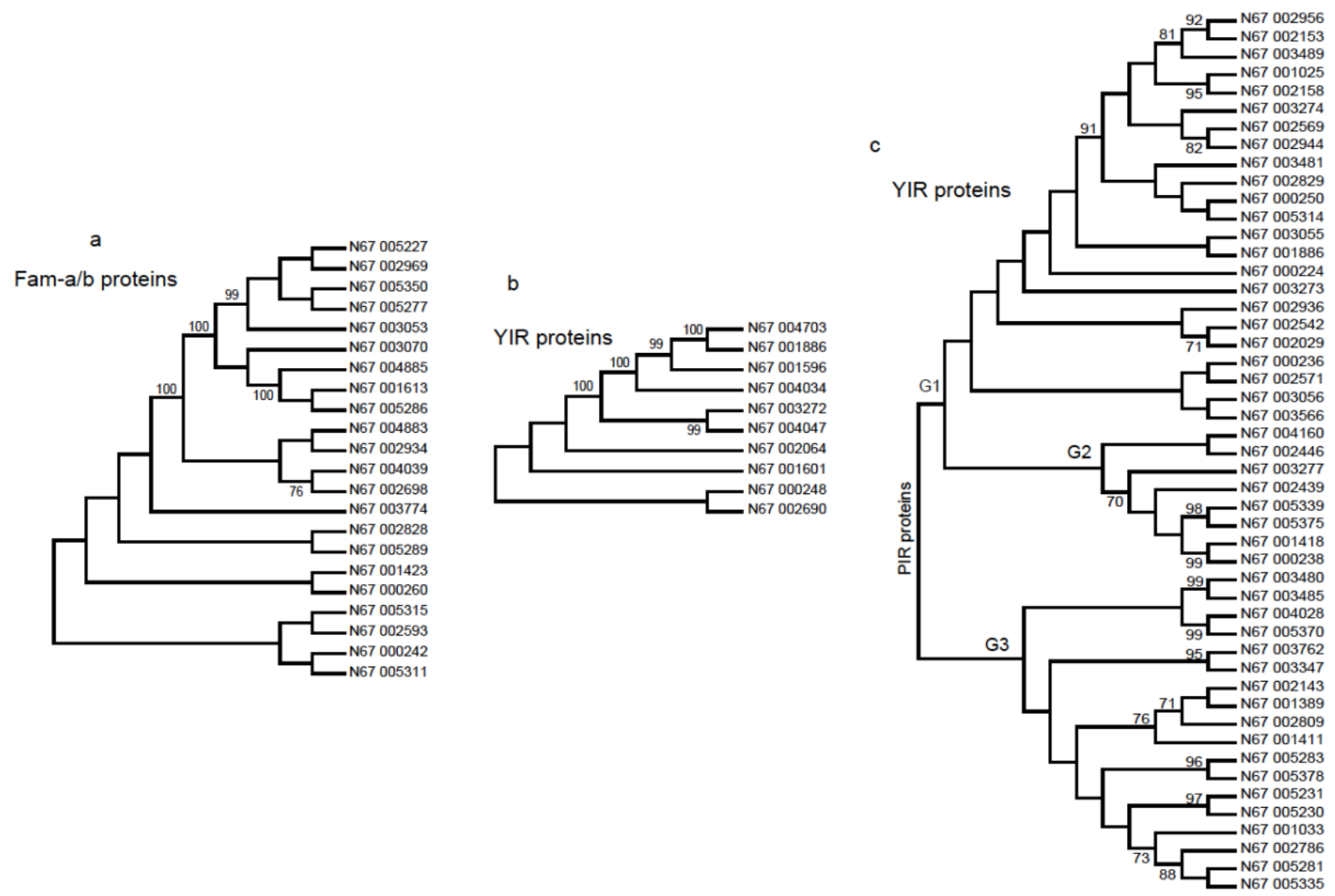

265 Fig. S2. Clustering of protein sequences from the Plasmodium y. nigeriensis N67-specific

266 orthogroups and those that are not assigned to any orthogroup. The predicted protein sequences

267 were aligned using ClustalW algorithm and clustered using procedures described in the Methods

268 section. a, Fam-A/B proteins; b, YIR proteins (group 1); c, YIR proteins (group 2). Only

269 bootstrap values higher than $70 \%$ are shown.

271 Gene families from three $P$. yoelii parasites

272 Among the predicted genes and proteins, we identified 22 gene families that have been

273 previously found in Plasmodium yoelii [35] with at least one member detected in N67 (Table

274 S10). The gene families consist of 1,475 genes ( $24 \%$ of the predicted genes) for $17 \mathrm{X}, 1,141$ 
275 genes (21\%) for N67, and 1,075 genes (19\%) for YM parasite (Table S10). The largest gene 276 families are the $y \mathrm{ir}$ and $\mathrm{fam}-\mathrm{a} / \mathrm{b} / \mathrm{c} / \mathrm{d}$ families. There are 1,057 yir and $301 \mathrm{fam}-\mathrm{a} / \mathrm{b} / \mathrm{c} / \mathrm{d}$ genes for 277 17X, 750 yir and 213 fam-a/b/c/d genes for N67, and 773 yir and $190 \mathrm{fam}-\mathrm{a} / \mathrm{b} / \mathrm{c} / \mathrm{d}$ genes for YM 278 parasite, respectively. As expected, clustering of YIR and Fam-A protein families showed that 279 the proteins from N67 grouped separately from those of 17X and YM (Fig. 3a and Fig. 3b), 280 consistent with N67 being a subspecies of $P$. yoelii. The true numbers of $y i r$ and $f a m-a / b / c / d$ 281 genes for the N67 and YM parasites could be larger because some genes in these gene families 282 are likely not assembled into the genome. Other important gene families include genes encoding 283 early transcribed membrane proteins (ETRAMPs), lysophospholipase, erythrocyte membrane 284 antigens, and reticulocyte binding proteins associated with host-parasite interactions and Cys6 285 (6-Cysteine) proteins. As noted above, there are 83 copies of the gene encoding PcEMA1 286 homologs [29] in the N67 parasite, compared with only one gene in the 17X and YM parasites, 287 respectively (Table S10). Clustering of the PcEMA1 proteins from the three parasites showed 288 that N67_000859 and N67_000245 were closely related to the two single copies from 17X 289 (17X_10019001) and YM (YM_100119001) (Fig. 4). Similarly, there are nine genes encoding 290 haloacid dehalogenase-like hydrolase in N67, but only 4 genes in both 17X and YM parasites. In 291 contrast, the number of genes encoding reticulocyte binding proteins appears to be reduced in 292 N67 parasites; 12 genes for N67 (including five from N67 specific orthogroups), whereas 17X 293 and YM have 33 and 31, respectively. Although we cannot rule out that the differences in gene 294 number are due to different degrees of genome completeness, the increases in gene number of 295 PcEMA1 homolog and haloacid dehalogenase-like hydrolase in N67 could be associated with its 296 unique growth characteristics and stimulation of strong early type I interferon response [13]. The 297 expansion of the PcEMA1 homolog genes deserve additional investigation. 

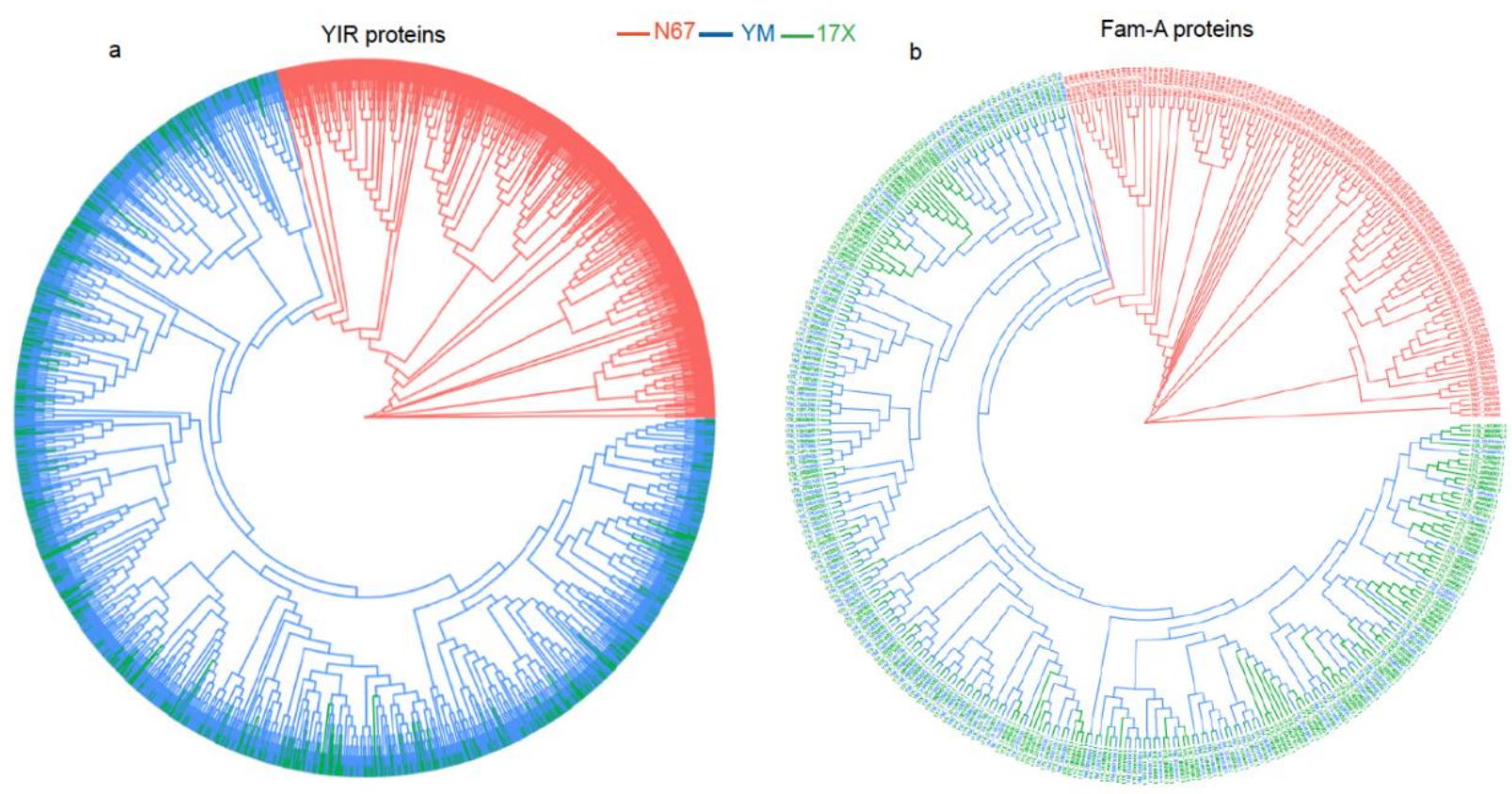

299 Fig. 3. Clustering of YIR and Fam-A proteins from the Plasmodium y. nigeriensis N67, P. y.

300 yoelii $17 \mathrm{X}$, and $P$. y. yoelii YM parasites. The predicted protein sequences were aligned using

301 ClustalW algorithm in msa $\mathrm{R}$ package, and the dendrograms were inferred and visualized using

302 the ape, seqinr, and ggtree packages in R. a, YIR proteins from N67, 17X, and YM parasites; b,

303 Fam-A proteins. Proteins are colored based on their parasite origins. 


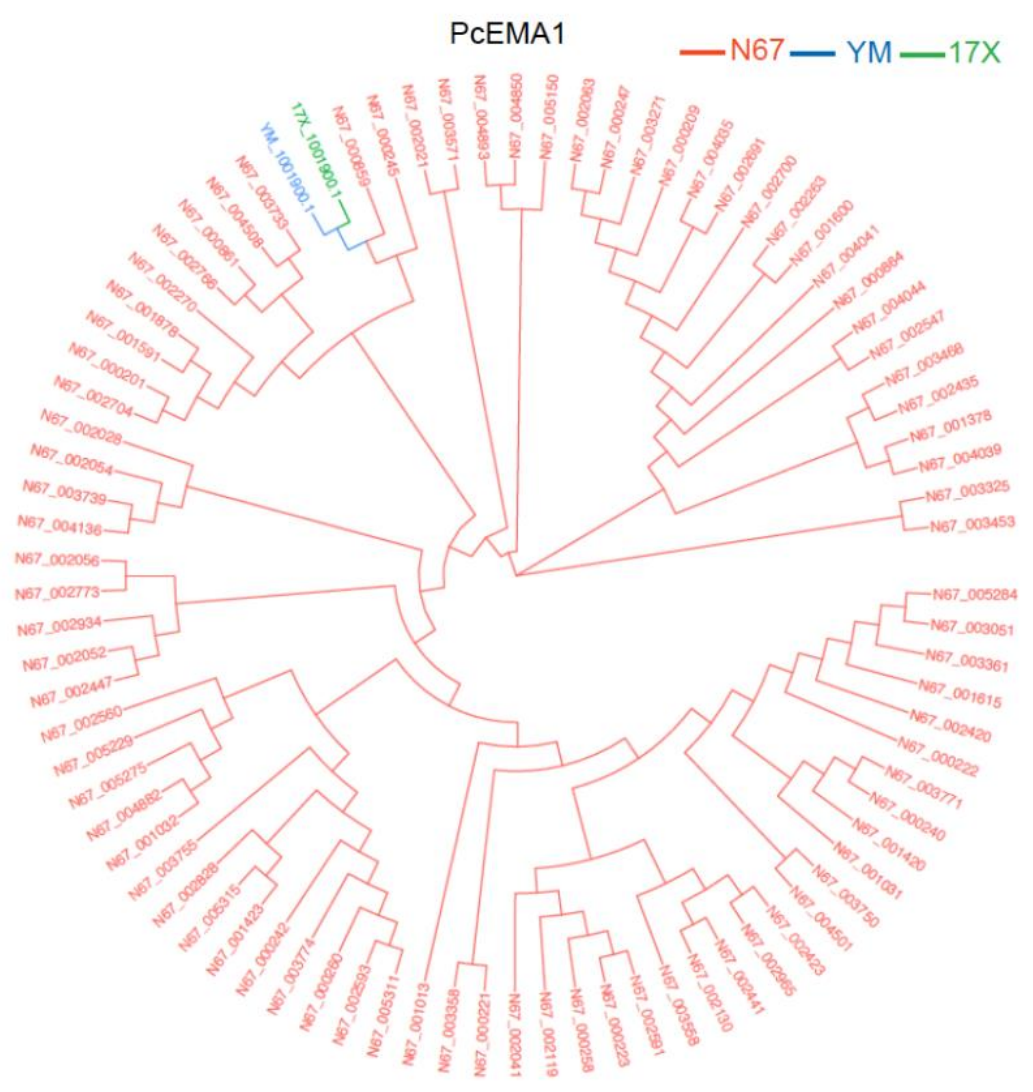

306 Fig. 4. Clustering of homologous $P$. chabaudi erythrocyte membrane protein 1 (PcEMA1) from

307 Plasmodium y. nigeriensis N67, P. y. yoelii 17X and P. y. yoelii YM parasites. The gene family

308 is expanded only in the N67 parasite. The predicted protein sequences were aligned using

309 ClustalW algorithm in msa $\mathrm{R}$ package, and the dendrogram was inferred and visualized using the

310 ape, seqinr, and ggtree packages in R. Proteins are colored based on their parasite origins.

\section{Sequence polymorphisms between N67 and 17X}

313 Initial alignment of the N67 sequences to those of 17X identified 486,102 SNPs and 41,317

314 indels, leading to approximately one SNP per $47.3 \mathrm{bp}$ and one indel per $556.7 \mathrm{bp}$ DNA between

$31517 \mathrm{X}$ and N67 assuming a genome size of $23 \mathrm{Mb}$ [18]. The number of SNPs of this study are

316 similar to the previous 458,922 SNPs from Illumina reads [6]. We further filtered the SNPs using

317 Ensembl Variant Effect Predictor (VEP) based on the impact of the variants on the protein 
318 function and the following criteria: coverage in both strains with a minimum depth of $5 \mathrm{X}$ and a 319 dominant allele frequency of $75 \%$. We identified 69,413 SNPs and 11,076 indels that passed the

320 following criteria and had high or moderate impacts (Table S11). These variants represent

321 approximately one SNP per 331.4 bp and one indel per 2076.6 bp of DNA. High impact variants

322 are those assumed to be disruptive on the protein functions such as total loss of function caused

323 by protein truncation. Moderate impact variants are non-disruptive variants that might change

324 protein effectiveness such as missense mutations, in-frame indels, and splice-region variants

325 outside the canonical splice site

326 (http://uswest.ensembl.org/info/genome/variation/prediction/predicted_data.html). Given that

327 there are 80,489 and 79,946 high or moderate impact variants between N67 vs 17X and YM,

328 respectively (Table S11), it is quite interesting that with so many differences between the

329 genomes, the YM (or 17XNL) and N67 parasites could be genetically crossed without apparent

330 difficulty in terms of mating or genetic crossover [5, 36].

332 Discussion

333 This study reports an annotated genome for the N67 parasite, a subspecies of $P$. yoelii

334 nigeriensis, using PacBio long sequence reads and RNA-Seq sequences from blood stage

335 parasites. The genomes of several $P$. yoelii strains belonging to different subspecies $(P . y$. yoelii),

336 including 17X, 17XNL, and YM strains, have been reported and well-characterized $[16,18,20]$.

337 Although Illumina-based RNA and DNA sequencing of the N67 parasite have been performed

338 for studying genetic polymorphisms and parasite response to drugs [6, 19], assembly of the N67

339 genome and prediction of gene functions have not been reported previously due to difficulties in

340 assembling AT-rich short sequence reads. Our study predicted and annotated 5,383 genes for 
341 N67 and identified a set of proteins with N67-specific orthology. Approximately 14\% of the

342 assembled N67 sequences did not align to the 17X genome. The unaligned sequences were likely

343 due to high level of sequence diversity between the 17X and N67 genomes and the limitations of

344 the assembling process. There are more than 80,000 SNPs and indels having medium to high

345 functional imacts betwen the N67 and 17X genomes. Improvement of the N67 genome with

346 increased overall alignment with the 17X reference would likely increase the numbers of SNPs

347 and indels. Interestingly, despite having highly diverse genomes (the level of diversity is greater

348 than human and chimpanzee), the N67 and YM or 17XNL can be genetically crossed to produce

349 progenies for mapping parasite and host genes [5, 36, 37]. The genome and transcriptome

350 sequences and annotations of the N67 parasite we present here will be valuable resources for

351 future studies on gene functions of this important $P$. yoelii subspecies.

353 The majority of studies using $P$. yoelii parasites involved the lethal YM (or 17XL) and nonlethal

354 17X (17XNL) strains. However, P. y. nigeriensis parasites have also been used as models for

355 studying unique disease phenotypes, transmission in mosquitoes, strain-specific host immune

356 responses and drug resistances [13, 14, 38-41]. The N67 and N67C are two isogenic strains of $P$.

$357 y$. nigeriensis subspecies that cause very unique and interesting disease phenotypes in C57BL/6

358 mice. Whereas mice infected with N67 parasites produce an early peak of IFN-I that has been

359 linked to suppression of parasitemia day 6 pi, mice infected with N67C produce low levels of

360 IFN-I and die approximately day 7 pi due to T cell and IFN- $\gamma$ mediated inflammation [13, 14]. A

361 mutation (C741Y) in PyEBL partially contributes to the difference in disease phenotypes [15];

362 however, other genes in the genome are likely involved, particularly the variant gene families

363 such as the $y i r$ and $f a m-a / b / c / d$ genes. For example, a locus at one end of chromosome 13 was 
364 shown to be significantly linked to many host genes functioning in IFN-I response pathways or

365 interferon-stimulated genes (ISGs) [37]. Among the proteins in Reactome pathways that may

366 play a role in viral mRNA translation and immune responses, several proteins can bind RNA and

367 DNA or have endopeptidase activities. These proteins may function to activate NFkB signaling,

368 ISG15 antiviral mechanism, and DDX58/IFIH1-mediated induction of IFN- $\alpha / \beta$ if they are

369 secreted into the host cytoplasm, particularly in liver cells. Further experiments are required to

370 demonstrate whether any of these proteins can influence host immune responses.

372 Approximately $20 \%$ of the annotated genes in the $P$. yoelii genomes are members of multi-copy

373 gene families. The number of the yir genes in 17X is much larger than that of N67 or YM.

374 Although the observation of more yir genes in the 17X genome could be due to more complete

375 and better annotated $17 \mathrm{X}$ genome, it is not surprising to find variation in the number of yir genes.

376 The yir genes belongs to the Plasmodium interspersed repeat (pir) gene families. The pir genes

377 are mostly distributed in the subtelomeric regions of chromosomes with gene copies numbering

378 from a few dozen to hundreds or even over a thousand [42], and up to $40 \%$ of the cir gene (gene

379 family from P. chanbaudi) repertoire are expressed during the intraerythrocytic cycle [43]. Many

380 of the pir genes are expressed on the surface of iRBCs and merozoites and play an important role

381 in immune evasion $[44,45]$. In addition to the yir genes, the variation in number of genes

382 encoding PcEMA1 homologs, haloacid dehalogenase-like hydrolases, reticulocyte binding

383 proteins between N67 and 17X/YM are interesting, particularly the expansion of the gene

384 encoding PcEMA1 homologs. These proteins likely play some important roles in host-parasite

385 interaction and parasite development. 


\section{Conclusions}

388 The rodent parasite N67 is an important subspecies of $P$. yoelii for studying host immune

389 responses and parasite biology. The lack of the assembled genome and genome annotation has

390 impeded functional studies of the parasite, including genetic mapping determinants playing

391 important roles in modulating host IFN-I responses. This study provides the first assembled and

392 annotated N67 parasite geneme, including prediction of 5,383 genes, although there are still

393 many gaps in the genome. Comparison of the annotated genome of the N67 parasite with those

394 of YM and 17X parasites reveals a large numbers of SNPs and indels that may have functional

395 impact on parasite development and biology. Additionally, unique N67 gene sets, expansion of

396 gene families, and genes potentially regulating host immune responses are also identified.

397 Although further efforts such as manual curation are necessary to completely assemble the

398 genome sequences, the assembled genome with over 5,000 predicted genes from this study will

399 greatly facilitate our investigations of the parasite biology and mechanisms of the disease.

\section{Methods}

\section{Parasite and infection of mice}

403 The N67 parasite was initially obtained from MR4-BEI

404 (https://www.beiresources.org/About/MR4.aspx) and were described previously (26). Inbred

405 female C57BL/6 mice, aged 6-8 weeks old, were obtained from NIAID/Taconic repository. The

406 procedures for infecting mice with the parasites were as reported previously $(26,27)$. Parasitemia

407 was monitored by microscopic examination of Giemsa-stained thin blood smears.

408

409

\section{DNA preparation for PacBio sequencing}


410 Mice were injected ip with an inoculum containing $1 \times 10^{6}$ infected red blood cells (iRBCs).

411 Blood samples $(200 \mu \mathrm{l})$ with approximately 30-40\% parasitemia were collected on day 4 after

412 injection. Infected red blood cells collected in $1 \mathrm{ml} 0.15 \%$ sodium citrate/PBS buffer were

413 pelleted at 2,000 rpm for $5 \mathrm{~min}$ in an Eppendorf centrifuge, re-suspended in $1 \mathrm{ml}$ of PBS, and

414 passed through two consecutive NWF filters (Zhixing Bio, Bengbu, China) to remove the host

415 white blood cells [19]. The flow-through cell suspension was washed in $800 \mu$ PBS $3 \mathrm{X}$ through

416 centrifugation at 3,000 rpm for $3 \mathrm{~min}$. The pellet was dissolved in $400 \mu \mathrm{l}$ lysis buffer $(100 \mathrm{mM}$

$417 \mathrm{NaCl}, 10 \mathrm{mM}$ Tris, $25 \mathrm{mM}$ EDTA, pH 8.0, 0.5\% SDS $)$ containing $20 \mu \mathrm{l}$ Rase $(500 \mu \mathrm{g} / \mathrm{ml})$ and

$41820 \mu \mathrm{l}$ protease $\mathrm{K}(10 \mathrm{mg} / \mathrm{ml})$ and incubated at $50^{\circ} \mathrm{C}$ overnight. DNA was extracted using $400 \mu \mathrm{l}$

419 phenol, chloroform, and isopropanol at a ratio of 25:24:1 and precipitated by adding 2 volume

$420100 \%$ ethanol overnight at $-20^{\circ} \mathrm{C}$. The sample was centrifuged at $13,000 \mathrm{rpm}$ for $15 \mathrm{mins}$ at $4^{\circ} \mathrm{C}$,

421 and the DNA pellet was washed in $500 \mu 170 \%$ ethanol twice before addition of $20 \mu l$ water. The

422 quality of DNA was estimated on 1\% agarose gel showing a typical high molecular weight band.

423

424 Fragmentation of DNA and PacBio sequencing

425 A SMRTbell library was constructed using standard PacBio library preparation procedure

426 (Pacific Biosciences, Menlo Park, CA, USA). The genomic DNA was fragmented with the

427 majority of DNA fragments above $20 \mathrm{~kb}$, then the DNA was carried into the first enzymatic

428 reaction to remove single-stranded overhangs and tailed with an A-overhang. Ligation with T-

429 overhang SMRTbell adapters was performed and the SMRTbell library was purified. The size

430 and concentration of the final library were assessed. 
432 Sequencing primer and Sequel DNA Polymerase were annealed and bound, respectively, to the

433 SMRTbell library. The library was loaded on PacBio Sequel using diffusion loading. SMRT

434 sequencing was performed on the Sequel System with Sequel Sequencing Kit 3.0, 1200 min

435 movies. Quality control (QC) for raw reads (subreads) generated from the sequencer were

436 performed by the default smrtlink QC pipeline. Pass-filter reads were then used as input for the

437 genome assembly.

\section{Genome sequence assembly}

440 The genome was assembled using HGAP v4.0, a standard assembler from PacBio SMRTLink

441 software (Pacific Biosciences, Menlo Park, CA, USA), Subreads longer than 6 kb were

442 designated as "seed reads" and used as template sequences for preassembly/error correction.

443 After assembly, two rounds of polishing were performed to increase the consensus sequence

444 quality of the assembly, including aligning the PacBio data to the contigs and computing

445 consensus using the Arrow consensus caller (SMRTLink).

\section{RNA-Seq, transcriptome assembly and gene predictions}

448 To assemble the N67 transcriptome, we extracted RNA from mixed-stage iRBC samples of eight

449 mice infected with N67 and performed Illumina sequencing as reported previously [15]. The

450 resulting RNA-Seq reads were trimmed with Trimmomatic [46] to remove the adapter

451 sequences, and the reads were mapped to the $17 \mathrm{X}$ genome using the STAR aligner [47] and

452 disambiguate [48] tools. RNA-Seq reads from the samples uniquely mapped to the 17X genome

453 were retained for the transcriptome assembly step that was performed using Trinity [26]. 
454 Gene predictions were generated using the MAKER pipeline [28]. Specifically, MAKER utilized

455 BLAST to align the de novo assembled N67 transcriptome and 17X transcriptome to the de novo 456 assembled N67 genome, polished these alignments using Exonerate in a splice-aware fashion, 457 and implemented SNAP and Augustus hidden Markov models (HMMs) to generate ab initio 458 gene models [49, 50]. Functional analysis and annotation was performed with InterProScan [51] 459 after homology searches of over 15 databases including Pfam, ProSite, TIGRFAM, and 460 PANTHER. Our final set of N67 genes/proteins only include those that are adequately supported 461 by the assembled N67 genome/transcriptome and the 17X proteome. For each predicted N67 462 protein, we computed two AED values (AED/eAED: at the base pair and exon levels) to quantify 463 how well each N67 protein is supported by these data sources [52].

\section{Estimates of completeness}

466 We quantified the completeness of the de novo assembled N67 transcriptome, genome, and 467 proteome using BUSCO [53] against the single-copy orthologs conserved among Apicomplexa 468 (446 BUSCOs) and Plasmodium (3642 BUSCOs) from the OrthoDB v10.1 database [54].

\section{Identification of orthologs}

471 We used the Orthofinder [34] framework for identifying the ortholog sets among the 17X, YM 472 and N67 proteins. Orthofinder utilizes DIAMOND [33] for identifying sequence similarity and 473 DendroBLAST [55] for gene tree inference. 
476 Hierarchical clustering analyses were performed using MEGAX [56]. Protein sequences of YIR,

477 Fam-A as well as proteins from the N67-specific orthogroups and the ones not assigned to any

478 orthogroup were aligned using the ClustalW algorithm. The maximum likelihood method and

479 Jones-Taylor-Thornton (JTT) matrix-based model were used to construct cladograms from the

480 aligned sequences [57].

481

\section{Availability of data and materials}

483 This sequencing data and assembled genomes have been deposited to GenBank with Accession

484 number JAEVLW000000000.

485

486 Abbreviation

487 YM, Plasmodium y. yoelii YM

488 17X, Plasmodium y. yoelii 17X

489 17XNL, Plasmodium y. yoelii 17XNL

490 17XL, Plasmodium y. yoelii 17XL

$491 \quad$ N67, Plasmodium y. nigeriensis N67

492 IFN-I, type I interferon

493 PyEBL, $P$. yoelii erythrocyte binding-like protein

494 SNPs, single nucleotide polymorphisms

495 HGAP, hierarchical genome assembly process

496 CCS, circular consensus sequencing

497 AED, annotation edit distance

498 pi, post infection 
PcEMA1, P. chabaudi erythrocyte membrane protein 1

500 MMEJ, microhomology-mediated end joining

501 BUSCO, benchmarking universal single-copy orthologs

502 ETRAMPs, early transcribed membrane proteins

503 VEP, variant effect predictor

504 ISGs, interferon-stimulated genes

505 pir, interspersed repeat

506 yir, yoelii interspersed repeats

507 iRBCs, red blood cells

508 QC, Quality control

509 HMMs, augustus hidden markov models

510 LCB, co-linear block

\section{References}

513 1. WHO: World malaria report 2019. https://wwwwhoint/malaria/publications/world-

$514 \quad$ malaria-report-2019/en/2019.

515 2. Craig AG, Grau GE, Janse C, Kazura JW, Milner D, Barnwell JW, Turner G, Langhorne

$516 \mathrm{~J}$, participants of the Hinxton Retreat meeting on Animal Models for Research on Severe

517 M: The role of animal models for research on severe malaria. PLoS Pathog 2012,

$518 \quad$ 8(2):e1002401.

519 3. Langhorne J, Buffet P, Galinski M, Good M, Harty J, Leroy D, Mota MM, Pasini E, 520 Renia L, Riley E et al: The relevance of non-human primate and rodent malaria $521 \quad$ models for humans. Malar $J$ 2011, 10:23.

522 4. Li J, Zhang Y, Sullivan M, Hong L, Huang L, Lu F, McCutchan TF, Su XZ: Typing $523 \quad$ Plasmodium yoelii microsatellites using a simple and affordable fluorescent labeling 524 method. Mol Biochem Parasitol 2007, 155(2):94-102. 
5. Li J, Pattaradilokrat S, Zhu F, Jiang H, Liu S, Hong L, Fu Y, Koo L, Xu W, Pan W et al: Linkage maps from multiple genetic crosses and loci linked to growth-related virulent phenotype in Plasmodium yoelii. Proc Natl Acad Sci U S A 2011, 108(31):E374-382.

6. Nair SC, Pattaradilokrat S, Zilversmit MM, Dommer J, Nagarajan V, Stephens MT, Xiao W, Tan JC, Su XZ: Genome-wide polymorphisms and development of a microarray platform to detect genetic variations in Plasmodium yoelii. Mol Biochem Parasitol

7. Pattaradilokrat S, Cheesman SJ, Carter R: Congenicity and genetic polymorphism in cloned lines derived from a single isolate of a rodent malaria parasite. Mol Biochem

8. Pattaradilokrat S, Li J, Wu J, Qi Y, Eastman RT, Zilversmit M, Nair SC, Huaman MC,

9. Martin-Jaular L, Ferrer M, Calvo M, Rosanas-Urgell A, Kalko S, Graewe S, Soria G,

10. Bakir HY, Sayed FG, Rahman SA, Hamza AI, Mahmoud AE, Galal LA, Attia RA:

11. Wykes MN, Liu XQ, Beattie L, Stanisic DI, Stacey KJ, Smyth MJ, Thomas R, Good MF:

12. Otsuki H, Kaneko O, Thongkukiatkul A, Tachibana M, Iriko H, Takeo S, Tsuboi T, Torii

Plasmodium strain determines dendritic cell function essential for survival from malaria. PLoS Pathog 2007, 3(7):e96.

M: Single amino acid substitution in Plasmodium yoelii erythrocyte ligand determines its localization and controls parasite virulence. Proc Natl Acad Sci U S A 2009, 106(17):7167-7172.

et al: Strain-specific innate immune signaling pathways determine malaria 
parasitemia dynamics and host mortality. Proc Natl Acad Sci U S A 2014, 111(4):E511-520.

557 14. Lacerda-Queiroz N, Riteau N, Eastman RT, Bock KW, Orandle MS, Moore IN, Sher A, Long CA, Jankovic D, Su XZ: Mechanism of splenic cell death and host mortality in a

15. Peng YC, Qi Y, Zhang C, Yao X, Wu J, Pattaradilokrat S, Xia L, Tumas KC, He X, Ishizaki T et al: Plasmodium yoelii Erythrocyte-Binding-like Protein Modulates Host

16. Carlton JM, Angiuoli SV, Suh BB, Kooij TW, Pertea M, Silva JC, Ermolaeva MD, Allen JE, Selengut JD, Koo HL et al: Genome sequence and comparative analysis of the

17. Vaughan A, Chiu SY, Ramasamy G, Li L, Gardner MJ, Tarun AS, Kappe SH, Peng X: model rodent malaria parasite Plasmodium yoelii yoelii. Nature 2002, 419(6906):512-

18. Otto TD, Bohme U, Jackson AP, Hunt M, Franke-Fayard B, Hoeijmakers WA, Religa AA, Robertson L, Sanders M, Ogun SA et al: A comprehensive evaluation of rodent

19. Li J, Cai B, Qi Y, Zhao W, Liu J, Xu R, Pang Q, Tao Z, Hong L, Liu S et al: UTR introns, antisense RNA and differentially spliced transcripts between Plasmodium yoelii subspecies. Malar J 2016, 15:30.

21. Nakano K, Shiroma A, Shimoji M, Tamotsu H, Ashimine N, Ohki S, Shinzato M,

20. Kooij TW, Carlton JM, Bidwell SL, Hall N, Ramesar J, Janse CJ, Waters AP: A

22. Aurrecoechea C, Brestelli J, Brunk BP, Dommer J, Fischer S, Gajria B, Gao X, Gingle A, Plasmodium whole-genome synteny map: indels and synteny breakpoints as foci for species-specific genes. PLoS Pathog 2005, 1(4):e44.

Minami M, Nakanishi T, Teruya K et al: Advantages of genome sequencing by longread sequencer using SMRT technology in medical area. Hum Cell 2017, 30(3):149161.

Grant G, Harb OS et al: PlasmoDB: a functional genomic database for malaria parasites. Nucleic Acids Res 2009, 37(Database issue):D539-543. 
23. Li H: Minimap2: pairwise alignment for nucleotide sequences. Bioinformatics 2018, 34(18):3094-3100.

24. Darling AE, Mau B, Perna NT: progressiveMauve: multiple genome alignment with gene gain, loss and rearrangement. PLoS One 2010, 5(6):e11147.

25. Wingett SW, Andrews S: FastQ Screen: A tool for multi-genome mapping and quality control. F1000Res 2018, 7:1338.

592 26. Grabherr MG, Haas BJ, Yassour M, Levin JZ, Thompson DA, Amit I, Adiconis X, Fan 593 L, Raychowdhury R, Zeng Q et al: Full-length transcriptome assembly from RNASeq data without a reference genome. Nat Biotechnol 2011, 29(7):644-652.

595 27. Langdon WB: Performance of genetic programming optimised Bowtie2 on genome

28. Campbell MS, Holt C, Moore B, Yandell M: Genome Annotation and Curation Using MAKER and MAKER-P. Curr Protoc Bioinformatics 2014, 48:4 11 11-39.

29. Deleersnijder W, Prasomsitti P, Tungpradubkul S, Hendrix D, Hamers-Casterman C,

30. Xu R, Liu Y, Fan R, Liang R, Yue L, Liu S, Su XZ, Li J: Generation and functional characterisation of Plasmodium yoelii csp deletion mutants using a microhomologybased CRISPR/Cas9 method. Int J Parasitol 2019, 49(9):705-714.

31. Hakimi MA, Bougdour A: Toxoplasma's ways of manipulating the host transcriptome via secreted effectors. Curr Opin Microbiol 2015, 26:24-31.

611 33. Buchfink B, Xie C, Huson DH: Fast and sensitive protein alignment using DIAMOND. Nat Methods 2015, 12(1):59-60.

613 34. Emms DM, Kelly S: OrthoFinder: phylogenetic orthology inference for comparative genomics. Genome Biol 2019, 20(1):238. 
$61535 . \quad$ Frech $\mathrm{C}$, Chen $\mathrm{N}$ : Variant surface antigens of malaria parasites: functional and 616 evolutionary insights from comparative gene family classification and analysis. $B M C$ $617 \quad$ Genomics 2013, 14:427.

618 36. Nair SC, Xu R, Pattaradilokrat S, Wu J, Qi Y, Zilversmit M, Ganesan S, Nagarajan V, 619 Eastman RT, Orandle MS et al: A Plasmodium yoelii HECT-like E3 ubiquitin ligase regulates parasite growth and virulence. Nat Commun 2017, 8(1):223.

621 37. Wu J, Cai B, Sun W, Huang R, Liu X, Lin M, Pattaradilokrat S, Martin S, Qi Y, Nair SC et al: Genome-wide Analysis of Host-Plasmodium yoelii Interactions Reveals Regulators of the Type I Interferon Response. Cell Rep 2015, 12(4):661-672.

39. Orfano AS, Duarte AP, Molina-Cruz A, Pimenta PF, Barillas-Mury C: Plasmodium yoelii nigeriensis (N67) Is a Robust Animal Model to Study Malaria Transmission by

40. Beaute-Lafitte A, Altemayer-Caillard V, Chabaud AG, Landau I: Plasmodium yoelii nigeriensis: biological mechanisms of resistance to chloroquine. Parasite 1994,

41. Graves PM, Curtis CF: Susceptibility of Anopheles gambiae to Plasmodium yoelii nigeriensis and Plasmodium falciparum. Ann Trop Med Parasitol 1982, 76(6):633-639. Cunningham D, Lawton J, Jarra W, Preiser P, Langhorne J: The pir multigene family of Plasmodium: antigenic variation and beyond. Mol Biochem Parasitol 2010, 170(2):65-73.

Lawton J, Brugat T, Yan YX, Reid AJ, Bohme U, Otto TD, Pain A, Jackson A, Berriman $\mathrm{M}$, Cunningham $\mathrm{D}$ et al: Characterization and gene expression analysis of the cir multi-gene family of Plasmodium chabaudi chabaudi (AS). BMC Genomics 2012, 13:125.

44. Yam XY, Brugat T, Siau A, Lawton J, Wong DS, Farah A, Twang JS, Gao X, Langhorne J, Preiser PR: Characterization of the Plasmodium Interspersed Repeats (PIR) proteins of Plasmodium chabaudi indicates functional diversity. Sci Rep 2016, 6:23449. 
646 45. Yam XY, Preiser PR: Host immune evasion strategies of malaria blood stage 647 parasite. Mol Biosyst 2017, 13(12):2498-2508.

648 46. Bolger AM, Lohse M, Usadel B: Trimmomatic: a flexible trimmer for Illumina 649 sequence data. Bioinformatics 2014, 30(15):2114-2120.

650 47. Dobin A, Davis CA, Schlesinger F, Drenkow J, Zaleski C, Jha S, Batut P, Chaisson M, 651 Gingeras TR: STAR: ultrafast universal RNA-seq aligner. Bioinformatics 2013, 652 29(1):15-21.

653 48. Ahdesmaki MJ, Gray SR, Johnson JH, Lai Z: Disambiguate: An open-source application for disambiguating two species in next generation sequencing data from grafted samples. F1000Res 2016, 5:2741.

656 49. Korf I: Gene finding in novel genomes. BMC Bioinformatics 2004, 5:59.

657 50. Stanke M, Steinkamp R, Waack S, Morgenstern B: AUGUSTUS: a web server for gene 658 finding in eukaryotes. Nucleic Acids Res 2004, 32(Web Server issue):W309-312.

659 51. Finn RD, Attwood TK, Babbitt PC, Bateman A, Bork P, Bridge AJ, Chang HY, 660 Dosztanyi Z, El-Gebali S, Fraser M et al: InterPro in 2017-beyond protein family and 661 domain annotations. Nucleic Acids Res 2017, 45(D1):D190-D199.

662 52. Eilbeck K, Moore B, Holt C, Yandell M: Quantitative measures for the management 663 and comparison of annotated genomes. BMC Bioinformatics 2009, 10:67.

664 53. Waterhouse RM, Seppey M, Simao FA, Manni M, Ioannidis P, Klioutchnikov G, 665 Kriventseva EV, Zdobnov EM: BUSCO Applications from Quality Assessments to 666 Gene Prediction and Phylogenomics. Mol Biol Evol 2018, 35(3):543-548.

667 54. Kriventseva EV, Kuznetsov D, Tegenfeldt F, Manni M, Dias R, Simao FA, Zdobnov 668 EM: OrthoDB v10: sampling the diversity of animal, plant, fungal, protist, bacterial and viral genomes for evolutionary and functional annotations of orthologs. Nucleic

671 55. Kelly S, Maini PK: DendroBLAST: approximate phylogenetic trees in the absence of 672 multiple sequence alignments. PLoS One 2013, 8(3):e58537.

673 56. Kumar S, Stecher G, Li M, Knyaz C, Tamura K: MEGA X: Molecular Evolutionary 674 Genetics Analysis across Computing Platforms. Mol Biol Evol 2018, 35(6):1547-1549. 675 57. Jones DT, Taylor WR, Thornton JM: The rapid generation of mutation data matrices 676 from protein sequences. Comput Appl Biosci 1992, 8(3):275-282. 
678 The authors thank Brigit Shea Sullivan, NIH Library Editing Service, for manuscript editing

679 assistance. We alos thank Dr. Jian Li, Xiamen University of China, for providing the NWF

680 filters.

681

682 Funding

683 This work was supported by the Division of Intramural Research, National Institute of Allergy

684 and Infectious Diseases (NIAID), National Institutes of Health (NIH), USA.

685

686 Author information

687 Affiliations

688 Malaria Functional Genomics Section, Laboratory of Malaria and Vector Research,

689 National Institute of Allergy and Infectious Disease, National Institutes of Health,

690 Bethesda, MD 20892-8132, USA

691 Cui Zhang, Lu Xia, Jian Wu, Yu-Chih Peng, Margaret Smith, Carole A. Long \& Xin-zhuan Su

692

693 NIAID Collaborative Bioinformatics Resource (NCBR), Frederick National Laboratory for

694 Cancer Research, Leidos Biomedical Research, Inc., Frederick, MD 21702, USA

695 Cihan Oguz, Sue Huse \& Justin Lack

696

697 Curret address: State Key Laboratory of Medical Genetics, Xiangya School of Medicine, 698 Central South University, Changsha, Hunan 410078, The People's Republic of China. 
$699 \quad \mathrm{Lu}$ Xia

700

701 The NCI sequencing facility, 8560 Progress Drive, Room 3007, Frederick Md 21701, USA

702 Jack Chen

703

704 Contributions

705 CZ, LX, Y-CP, JW, MS, parasite infection of mice, DNA preparation, data analysis; JC, PacBio

706 sequencing, $\mathrm{CO}, \mathrm{SH}, \mathrm{JL}$, data analysis and writing; CAL and X-zS supervision and writing; Xz-S

707 project conception.

708

709 Corresponding author

710 Correspondence to Xinzhuan Su.

711

\section{Ethics declarations}

713 Ethics approval and consent to participate

714 Plasmdoium yoelii nigeriensis N67 is a malaria parasite that infects red blood cells of rodents,

715 including mice. This study involves infection of C57BL/6 mice with the parasite to obtain

716 parasite genomic DNA for sequencing. The experiments were performed in accordance with the

717 protocol approved (approval \#LMVR11E) by the Institutional Animal Care and Use Committee

718 (IACUC) at the National Institute of Allergy and Infectious Diseases (NIAID) following the

719 guidelines of the Public Health Service Policy on Humane Care and Use of Laboratory Animals 
720 and The Association for Assessment and Accreditation of Laboratory Animal Care International

721 (AAALAC). The study was carried out in compliance with the ARRIVE guidelines.

722

723 Consent for publication

724 Not applicable.

725

726 Conflict of interests

727 The authors declare that they have no competing interests.

728

729 Supplementary information

730 Additional file 1. Fig. S1-2.

$731 \quad$ Additional file 2. Table S1.

732 Additional file 3. Table S2.

733 Additional file 4. Table S3.

$734 \quad$ Additional file 5. Table S4.

735 Additional file 6. Table S5.

736 Additional file 7. Table S6.

$737 \quad$ Additional file 8. Table S7.

$738 \quad$ Additional file 9. Table S8.

739 Additional file 10. Table S9.

740 Additional file 11. Table S10.

741 Additional file 12. Table S11. 


\section{Figures}

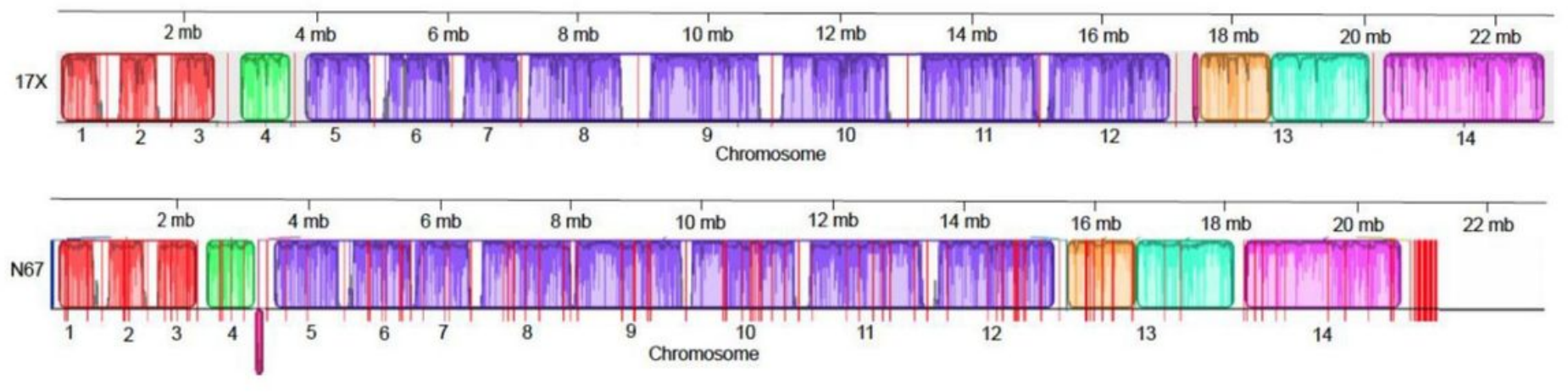

Figure 1

Alignment of Hierarchical Genome Assembly Process (HGAP) assembled N67 contigs to the 17X chromosomes. The alignments were generated using progressiveMauve. Each color corresponds to a localized co-linear block (LCB) that is conserved across the two genomes. Inside each LCB, the jagged dark lines represent the similarity profile; with darker colors representing higher similarity regions. The vertical red lines indicate chromosome boundaries in $17 \mathrm{X}$ and the contig boundaries on the N67 sequences. Note a contig on N67 chromosome 4 that is inverted (presented under the chromosome line) in reference to that of $17 \mathrm{X}$ sequence.
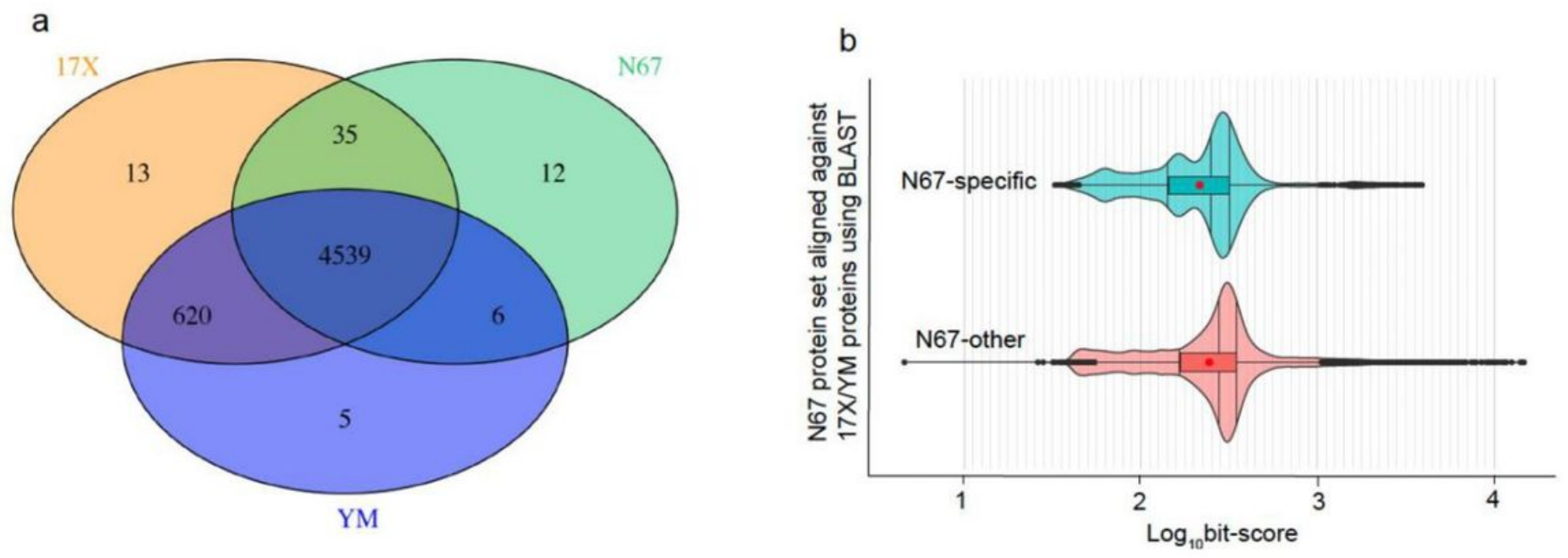

Figure 2

Shared and strain-specific orthogroup counts identified from Plasmodium y. yoelii YM, P. y. yoelii 17X, and P. y. nigeriensis N67 parasites using OrthoFinder [32]. a, Venn diagram of shared and strain-specific orthogroups; b, log10-transformed bit-score distributions for N67 proteins that are not assigned to any orthogroup plus those in N67-specific orthogroups (N67-specific) and proteins assigned to orthogroups having at least one 17X or YM protein (N67- other). The bit-scores are derived from pairwise BLAST 
alignments within the Orthofinder framework, where all queries were N67 protein sequences that were aligned against the 17X and YM sequences. The red dots indicate the mean values of bit-score distributions, whereas the vertical lines within the violins indicate the median, upper and lower quartile values.

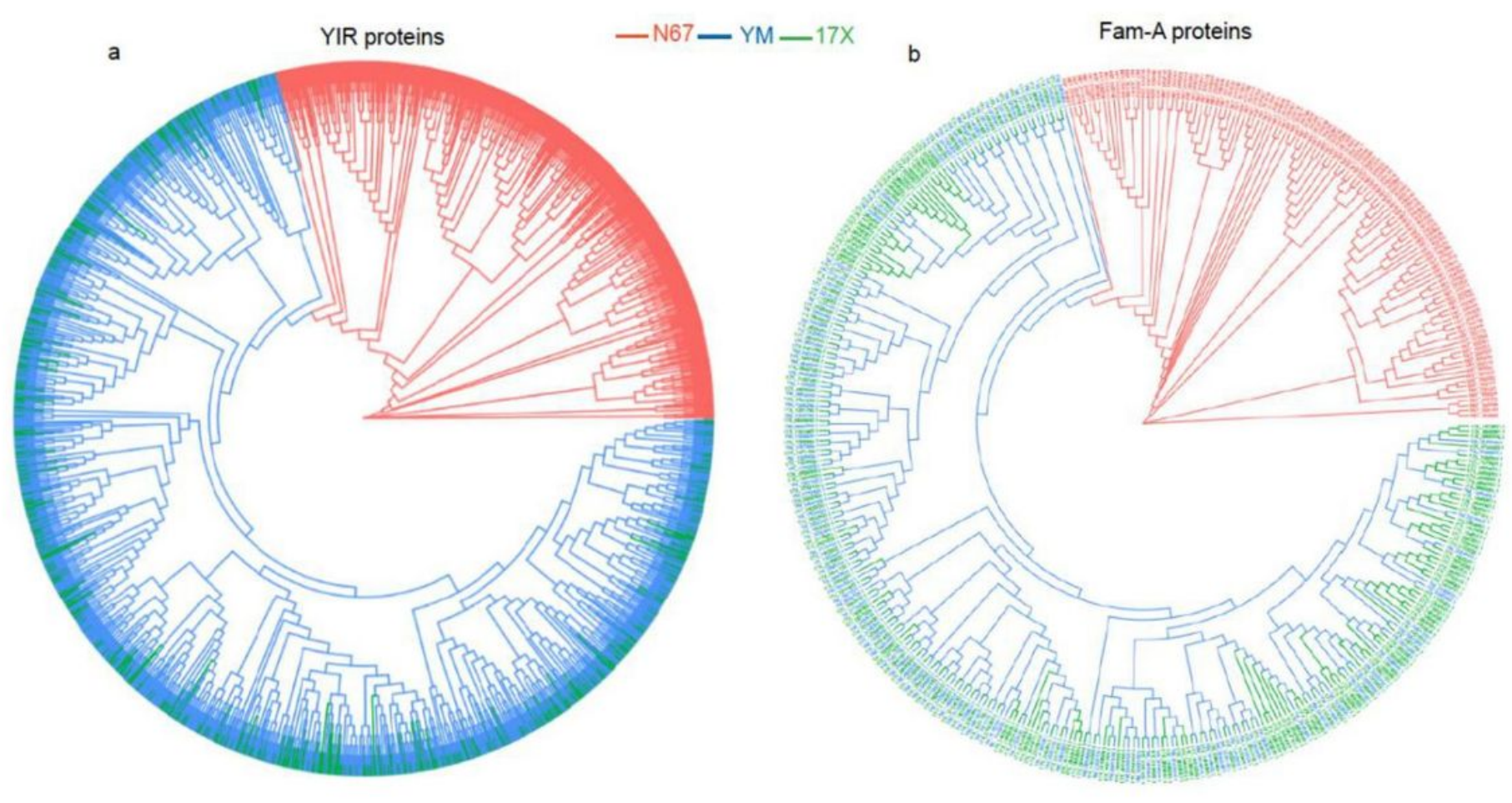

\section{Figure 3}

Clustering of YIR and Fam-A proteins from the Plasmodium y. nigeriensis N67, P. y. yoelii 17X, and P. y. yoelii YM parasites. The predicted protein sequences were aligned using ClustalW algorithm in msa $R$ package, and the dendrograms were inferred and visualized using the ape, seqinr, and ggtree packages in R. a, YIR proteins from N67, 17X, and YM parasites; b, Fam-A proteins. Proteins are colored based on their parasite origins. 


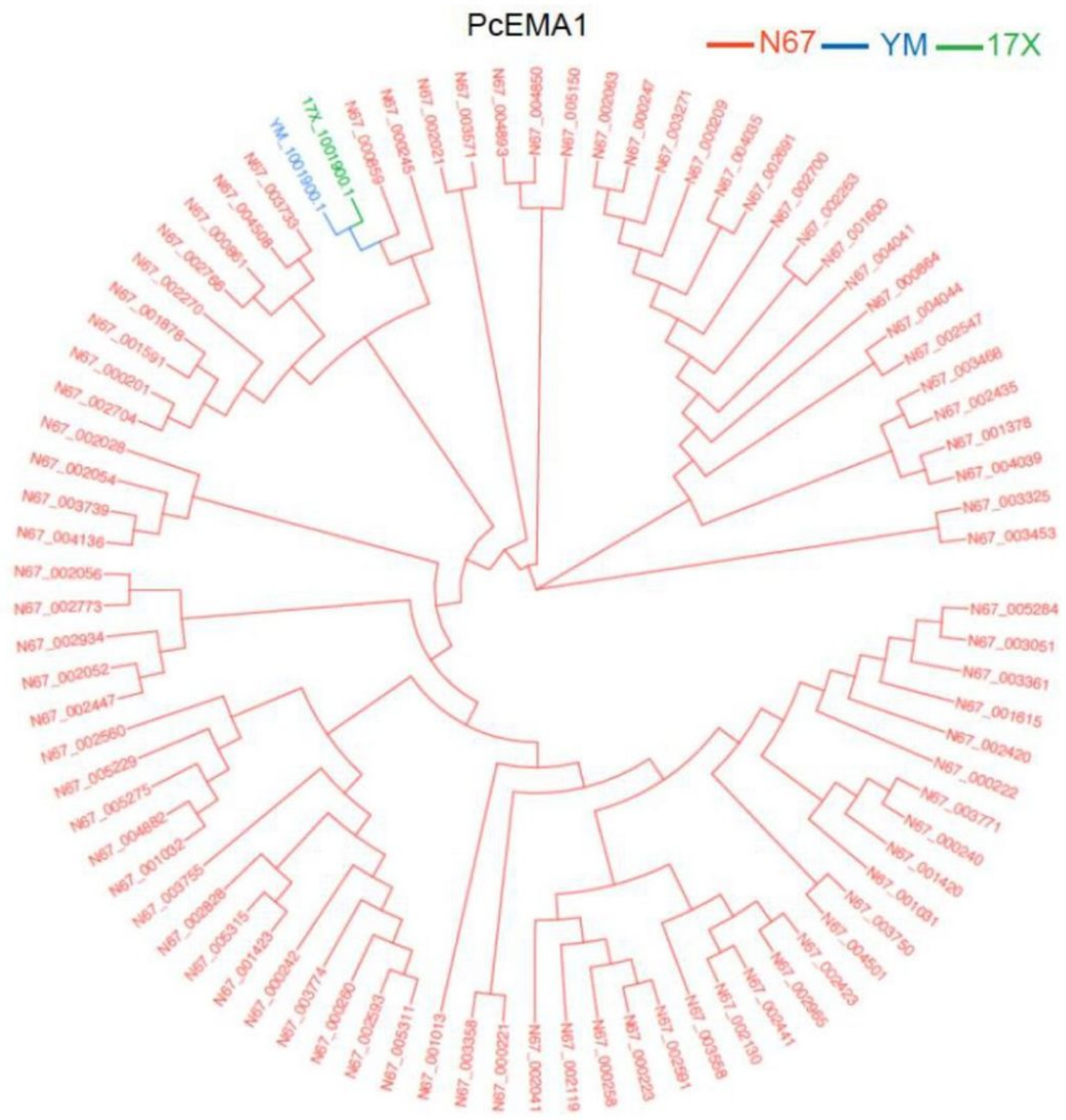

Figure 4

Clustering of homologous P. chabaudi erythrocyte membrane protein 1 (PcEMA1) from Plasmodium y. nigeriensis N67, P. y. yoelii 17X and P. y. yoelii YM parasites. The gene family is expanded only in the N67 parasite. The predicted protein sequences were aligned using ClustalW algorithm in msa R package, and the dendrogram was inferred and visualized using the ape, seqinr, and ggtree packages in R. Proteins are colored based on their parasite origins. 


\section{Supplementary Files}

This is a list of supplementary files associated with this preprint. Click to download.

- Table1.xlsx

- Table2.xlsx

- BNCN67Supplementalfiles.pdf

- TableS1.xlsx

- Tables10.xlsx

- TableS11..xlsx

- Tables2.xIsx

- TableS3.xIsx

- TableS4.xIsx

- TableS5.xIsx

- Tables6.xlsx

- TableS7.xIsx

- Tables8.xIsx

- Tables9.xIsx 